\title{
La vertu morale aristotélicienne en tant que médiété pros hemas : perspectives quantitative et relativiste
}

\section{Louise Rodrigue}

\section{(2) OpenEdition Journals}

Édition électronique

URL : https://journals.openedition.org/philosant/910

DOI : 10.4000/philosant.910

ISSN : 2648-2789

Éditeur

Éditions Vrin

\section{Édition imprimée}

Date de publication : 1 novembre 2013

Pagination : 243-264

ISBN : 978-2-7574-0591-8

ISSN : 1634-4561

Référence électronique

Louise Rodrigue, « La vertu morale aristotélicienne en tant que médiété pros hemas : perspectives quantitative et relativiste », Philosophie antique [En ligne], 13 | 2013, mis en ligne le 01 novembre 2018, consulté le 02 décembre 2022. URL : http://journals.openedition.org/philosant/910 ; DOI : https:// doi.org/10.4000/philosant.910

\section{cc) (1) $९$}

Creative Commons - Attribution - Pas d'Utilisation Commerciale - Pas de Modification 4.0 International - CC BY-NC-ND 4.0

https://creativecommons.org/licenses/by-nc-nd/4.0/ 


\section{LA VERTU MORALE ARISTOTÉLICIENNE \\ EN TANT QUE MÉDIÉTÉ PROS HEMAS : PERSPECTIVES QUANTITATIVE ET RELATIVISTE*}

Louise RODRIGUE

Collège universitaire dominicain, Ottawa

RÉSUMÉ. Cette étude porte sur la notion aristotélicienne de vertu morale dans son sens strict, c'est-à-dire entendue comme médiété fixée relativement à nous. L'analyse et la critique des interprétations dites « quantitatives » d'une part, « relativistes » d'autre part, résultent en une clarification de la définition donnée par les Éthiques (cf. 1106b36-1107a6 et 1227b8-9).

SUMMARY. This paper bears on Aristotelian moral virtue stricto sensu, which is the mean relative to us. By the critic review of the so-called quantitative and relativist readings, we shed light on the definition found in the Ethics (cf. 1106b36-1107a6 and 122768-9).

* Je remercie le Professeur Richard Bodéüs, qui a lu et commenté les différents états de ce texte, ainsi que le lecteur anonyme dont les remarques ont permis d'améliorer la version finale.

Philosophie antique, $\mathrm{n}^{\circ} 13$ (2013), 243-264 

Les versions nicomachéenne et eudémienne de la définition de la vertu morale identifient son genre comme hexis proairetike (1106b36; 1227b8). Or en raison de sa généralité, le genre ne renseigne pas suffisamment; dans la mesure où l'on conçoit la vertu seulement comme un état stable et permanent - même en précisant « décisionnel ${ }^{1}{ }^{~}-$, impossible de la distinguer du vice, puisque ce dernier se comprend également comme un état décisionnel ${ }^{2}$. C'est pourquoi Aristote affirme en 1106a14-15 qu'il ne faut pas «se borner à déclarer ainsi qu'elle [la vertu] est un état, mais encore indiquer quelle sorte d'état ». État de l'âme (désidérative) quant au genre, la vertu morale se distingue par la médiété, sa cause formelle ${ }^{3}$. Mais puisqu'il existe plusieurs types de milieu, Aristote précise qu'il s'agit de la

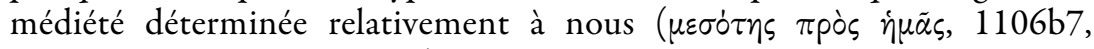
1106b36-1107a1 ; 1227b8-9). En tant que différence spécifique, la médiété pros hemas constitue l'élément central de la définition de la vertu morale ; à ce titre, cette notion a donné lieu à nombre de commentaires qui tentent d'éclaircir la nature de la vertu morale. Ces contributions se répartissent en deux groupes, selon leur perspective : les interprétations dites «quantitatives », qui insistent sur le concept de médiété, et les interprétations dites « relativistes », qui insistent sur l'aspect pros hemas. Les deux courants, complémentaires, expriment certaines vérités à propos de la vertu morale; la présente étude se propose de réunir dans une vision cohérente les acquis de chacun dans l'idée qu'une explicitation globale de la vertu en tant que médiété pros hemas se doit de les considérer ensemble, tout comme elle se doit de tenir compte des apports propres des deux traités de morale attribués au Stagirite, l'Éthique à Nicomaque (EN) et l'Éthique à Eudème $(E E)$. Après avoir écarté les exégèses les plus discutables, nous examinerons donc les explications de la médiété éthique de type quantitatif, puis celles

1. Pour un examen approfondi de l'aspect décisionnel de la vertu, voir Lorenz 2009.

2. Joachim 1951, p. 85-86; Urmson 1973, p. 223 ; Leighton 1995, p. 75 ; Broadie 2002, p. 306 ; Bodéüs 2004, p. 116 n. 2 ; Pakaluk 2005, p. 108.

3. Selon la terminologie employée par Gauthier et Jolif 1970, p. 144, et par Bosley 1991, p. 51. 
de type relativiste, démarche à l'issue de laquelle cette notion se trouvera clarifiée.

\section{Ce que la vertu n'est pas}

Afin que l'analyse de la vertu morale en tant que médiété pros hemas soit la plus fructueuse possible, il importe premièrement d'éliminer les interprétations manifestement erronées, qui ne tiennent pas compte de la richesse conceptuelle de la vertu présente dans les deux Éthiques. Si à l'évidence la vertu en tant que médiété ne saurait se confondre avec la médiocrité $^{4}$, ni avec la disposition morale inférieure qu'est la continence ${ }^{5}$, non plus qu'avec la moyenne arithmétique (1106b8), en revanche l'interprétation qui assimile la médiété à la modération constitue un écueil plus difficilement évitable. Bien que dénoncée comme fautive de façon convaincante $^{6}$, cette interprétation est encore reprise dans certains commentaires qui situent la médiété dans des actions et des affects modérés ${ }^{7}$. Selon ce point de vue, dans les circonstances où tel affect et telle action sont appropriés, la vertu consiste invariablement à éprouver l'affect en question et à agir de façon modérée : une personne douce manifestera la colère de façon modérée à chaque fois que cet affect se trouvera en jeu, un individu tempérant mangera et boira modérément, etc. Or, note J. O. Urmson, la modération est absurde ${ }^{8}$ dans certains cas : si par exemple l'injure est grande, la colère doit l'être tout autant - autrement dit, la colère du vertueux sera appropriée à la provocation, et non systématiquement modérée. L'explication d'Urmson en ce sens vise juste: Aristote définit la vertu morale comme un état moyen envers les affects et les actions, et non comme une disposition envers les affects et actions moyens ou intermédiaires'. Ajoutons à l'argument d'Urmson que, loin de s'opposer aux affects et actions d'une certaine ampleur, la médiété les implique parfois ;

4. En fait, elle en est tout le contraire puisque, dans l'ordre moral, la vertu constitue un extrême, un sommet; $c f .1107$ a7, 23. Voir Kenny 2011, p. xvii.

5. Celle-ci tient de celle-là, à cette différence près qu'elle implique une victoire sur ses propres affections ; $c f .1151 \mathrm{a} 26-27 ; 1223 \mathrm{~b} 12-14$.

6. Particulièrement par Urmson 1973 ; voir aussi Losin 1987, p. 333 ; Achtenberg 2002, p. 98-102; Pakaluk 2005, p. 109 ; Gottlieb 2009, p. 22-25; Lorenz 2009, p. 428; Wolf 2010, p. 74.

7. Voir par exemple Williams 1985, p. 36: « The theory oscillates between an unhelpful analytical model [...] and a substantively depressing doctrine in favor of moderation. The doctrine of the Mean is better forgotten [...] ». Cette position est rapportée comme erronée, injuste et insensible aux innovations aristotéliciennes par Losin 1987, p. 329, et Evrigenis 1999 , p. 396 ; voir aussi Gottlieb 2009, p. 19.

8. Urmson 1973, p. 225 ; il dit même « stupide ».

9. Idem; Young 1996 et Lawrence 2009, p. 428, reprennent ce même argument pour rejeter l'interprétation de la vertu-modération. 
pensons entre autres aux actions du magnifique, qui sont grandes tout en s'inscrivant dans la médiété $(1122 \mathrm{a} 23,11221 ;$ 1233a37), ce qui fait que celle-ci ne réside pas dans des dépenses moyennes, mais dans des dépenses qui conviennent ${ }^{10}$.

L'aspect pros hemas de la médiété éthique donne également lieu à certaines méprises interprétatives, répertoriées par Lesley Brown. Cette dernière s'emploie à dénoncer ce qui prend les allures d'une « dérive individualiste » dans le cadre de l'interprétation dire «relativiste » de la médiété ; l'auteure dénombre quatre espèces de relativité par rapport à l'agent moral inexactes. La première interprétation relie la variation de la médiété au niveau moral de l'agent : la vertu pour l'individu mature différerait de celle du débutant en termes d'exigence, en ce sens que les attentes seront plus élevées envers les agents moraux pleinement développés ${ }^{11}$. Même s'il est exact que la personne qui commence son entraînement moral n'est pas tenue au même résultat que la personne exercée à la vertu, il ne s'ensuit pas pour autant de fluctuation dans la vertu. Aristote est clair à cet effet : la vertu, le milieu, exclut l'excès et le défaut (1107a19-27); une chose telle que l'excès ou le défaut de moyenne n'existe pas, parce que la moyenne est une extrémité (voir note 4). La vertu constitue un sommet de perfection auquel, à l'image de l'œuvre réussie, on ne peut ni retrancher, ni ajouter quoi que ce soit (1106b9-15). De fait, la performance du débutant se situe hors de la vertu. Les Éthiques ne discutent pas la façon de «faire de son mieux » selon le niveau de développement moral de chacun; elles visent plutôt à définir l'excellence morale, ce qui précisément manque au novice. Du reste, Aristote ne cache pas la rareté de la vertu : elle n'est pas chose facile ni à la portée de tous (1109a24-29), ce qui explique qu'elle soit louable et belle (1109a29-30; 1233b16, 1234b14).

Le deuxième type d'erreur touchant l'interprétation relativiste de la médiété consiste à rendre la vertu fonction de la sensibilité affective de l'agent, de sorte que le courage, par exemple, différerait chez une personne timide et une personne audacieuse. Les tenants de cette position ${ }^{12}$ se basent sur l'observation, juste et conforme aux Éthiques, selon laquelle la constitution émotionnelle des agents rend l'atteinte de l'excellence plus ou moins facile. Brown concède qu'Aristote reconnaît la diversité des tempéraments incitant les individus à agir différemment; elle renvoie à 1109b1-7, où le Philosophe explique que chacun présente des inclinations naturelles dont il doit s'éloigner pour gagner le milieu (1109a12-19). Ajoutons EE,

10. Achtenberg 2002, p. 101.

11. Brown 1997, p. 82-83. L'auteure vise nommément la position de Leighton 1995, mais porte aussi atteinte au propos de Pakaluk 2005, p. 113, qui, sans tenir compte de l'objection, propose ce type d'explication.

12. La critique concerne surtout Losin 1987. 
1222a40 sqq., où l'inclination naturelle est aussi mentionnée, sauf que la « nature » en question est celle de l'espèce humaine et non de l'individu; Aristote y précise que la disposition la plus contraire au milieu se situe dans la direction vers laquelle la majorité des êtres humains est portée à errer ${ }^{13}$. Cela dit, admettre l'existence de penchants naturels, précise Brown, n’équivaut pas à soutenir que la médiété varie selon ceux-ci. Simplement les agents moraux atteignent la vertu à partir des différents points de départ que constituent leurs inclinations, certains plus difficilement, d'autres n'y parvenant pas du tout - comme l'atteste le phénomène de la continence ${ }^{14}$. Peu importent donc les données initiales - la constitution affective originelle-, puisque la destination demeure la même ; seul le parcours peut changer ${ }^{15}$.

Brown dispose troisièmement de l'interprétation se fondant sur Politiques 1260a20 sqq., passage d'après lequel la médiété varie en fonction du statut social. Dans cette perspective, la vertu se définirait différemment ou présenterait divers degrés - selon qu'on est un homme, une femme, un enfant, un esclave, un gouvernant, un gouverné, chacun devant faire preuve de courage, de tempérance, etc. de la façon convenant à son groupe social ${ }^{16}$. Brown concède qu'il s'agit de la conception de la vertu - et donc de la médiété - à l'œuvre dans les Politiques, mais souligne qu'elle ne concerne pas les Éthiques. La théorie politique vise la constitution d'une communauté, et pour ce faire doit tenir compte des nombreux rôles de ses membres afin de mieux les aménager et les harmoniser, alors que la théorie éthique tâche de définir la vie la meilleure pour un type de participant spécifique, le seul qui puisse vraiment y prétendre, l'homme adulte libre, intelligent, de bonne naissance et bien éduqué ${ }^{17}$. Ainsi l'excellence morale ne saurait fluctuer selon la condition sociale des agents, car une seule d'entre elles est en jeu dans l'éthique ${ }^{18}$. En fait, comme les Éthiques mettent l'accent sur la vie la meilleure, elles excluent d'emblée les individus qui, bien que dotés d'une

13. Cette remarque, contrairement à celle de l'EN, n'est assortie d'aucune recommandation, comme s'il appartenait au lecteur lui-même de déterminer ce qui est à faire, conférant à l' $E E$ une optique descriptive plutôt que normative.

14. Cet expédient de la vertu, plus commun que celle-ci, fait l'objet de remarques à l'occasion de l'examen de l'incontinence, au troisième livre commun aux deux Éthiques.

15. Brown 1997, p. 83-84.

16. Pakaluk 2005, p. 113, liste cette interprétation parmi les sortes de relativité qu'il qualifie de « raisonnables ».

17. Ce passage des Politiques donne lieu à la même conclusion chez Reeve 2012, p. 110111. Mais il n'est pas nécessaire de recourir aux Politiques pour saisir cette donnée ; l'EN exclut de l'excellence morale les enfants (qui vivent au gré de leurs appétits, 1119b5-6), les femmes et les gouvernés (en raison de leur infériorité, 1158b12-19; voir aussi 1238b16 sqq.), notant l'exigence, en regard du caractère, d'une certaine affinité avec la vertu (1179b30-31).

18. Brown 1997, p. 85. 
vertu ou excellence au sens politique du terme - c'est-à-dire une vertu au regard de leur fonction dans la collectivité -, n'ont pas accès à cette vie meilleure en raison de leur âge, leur sexe, leurs capacités, etc. ${ }^{19}$ Finalement, Brown réfute l'idée selon laquelle la médiété varie d'après les croyances individuelles : la manière appropriée d'être affecté et d'agir différerait d'un agent à l'autre selon ce que chacun croit à propos de la situation dans laquelle il se trouve (et ce même en cas de croyance erronée). L'auteure rappelle, pour éliminer cette option, qu'un individu agissant d'après une croyance (erronée) n'agit pas en connaissance de cause, alors que savoir ce que l'on fait constitue l'un des critères de l'excellence morale. La condition est énoncée en 1105a32 : l'agent doit savoir ce qu'il est en train d'exécuter pour que son acte soit vertueux; la même idée apparaît en $1225 \mathrm{~b} 5$ sqq. où Aristote précise qu'agir dans l'ignorance consiste à agir de façon involontaire, tandis que la vertu est délibérée.

\section{Les interprétations quantitatives de la médiété pros hemas}

Un tel exercice éliminatoire fournit certaines balises contribuant à déterminer ce qui, dans la première lignée interprétative, est recevable et ce qui l'est moins. Parmi les contributions de ce groupe, celle d'Urmson tient le haut du pavé, et est qualifiée de « quantitative » par d'autres exégètes ${ }^{20}$. C'est que l'auteur, dans sa présentation en cinq points de la doctrine de la médiété (exposée au livre II de chacune des Éthiques), accorde une grande importance à la notion de quantité. Il affirme, dans le premier point, qu’à chaque vertu morale particulière correspond une émotion - c'est ainsi qu'Urmson rend le terme pathos - qui en constitue le champ; dans le cas de chacune de ces émotions, il est possible d'être disposé de façon à en manifester le degré ou la quantité juste ${ }^{21}$, ce en quoi consiste la vertu ; il est possible, dans le cas de chaque émotion, d'être disposé de manière à en manifester soit trop, soit trop peu, chacune de ces dispositions étant un vice de caractère ; «trop ${ }^{22} »$, explique Urmson dans le quatrième point, inclut « dans un trop grand nombre d'occasions » aussi bien que « trop violemment », et « trop peu » inclut pour sa part «dans trop peu d'occasions » et d'autres possibilités comme « trop faiblement »; Urmson attribue finalement au Stagirite la position selon laquelle il n'existe pas d'émotion qu'un agent ne devrait pas manifester (elles sont toutes appropriées dans la me-

19. Pour une explication de ce qu'Aristote entend par « vertu des femmes et des esclaves » dans le cadre des Politiques, voir Deslauriers 2003.

20. Voir Curzer 1996, p. 129 ; Hursthouse 2005, p. 98 ; Pearson 2006, p. 81.

21. Urmson 1973, p. 226, utilise l'expression right amount.

22. On suppose ici qu'Urmson parle du trop et du trop peu mentionnés dans la précision par la négative que donne Aristote du milieu pros hemas, en $1106 a 32$. 
sure où l'on en manifeste une quantité adéquate). Cette brève synthèse de la doctrine de la médiété donnée par le commentateur révèle cependant certaines lacunes dans sa position. La critique qui concerne notre propos ${ }^{23}$ tient au fait qu'Urmson ne précise ni ne justifie le fondement de son interprétation, soit son identification de la médiété avec la quantité adéquate d'émotion - et ce d'autant plus que les Éthiques ne font pas explicitement cette association. Il note, conformément à $1106 \mathrm{~b} 8$, la différence entre le milieu pros hemas et le milieu mathématique ${ }^{24}$, mais sans plus, de sorte que le lecteur ne saisit pas vraiment comment la notion exprimée par l'expression right amount se distingue de la médiété de la seconde espèce, puisque la formule utilisée relève du domaine comptable et renvoie ainsi à une « masse » précise et fixe. Urmson observe, il est vrai, que la détermination de l'acte vertueux (donc de la quantité juste d'émotion à manifester) est fonction des facteurs propres à la situation dans laquelle l'agent se trouve ${ }^{25}$; néanmoins, il n'explicite pas non plus le rôle des circonstances dans la détermination de la vertu.

Avant de déployer cette difficulté, il importe de signaler que la locution right amount a été utilisée antérieurement à la contribution d'Urmson: H. H. Joachim adopte en effet une perspective quantitative dans son commentaire du livre II de l'Éthique à Nicomaque. Comparant l'action à une statue, il explique que, tout comme celle-ci est belle en raison du bon usage que fait le sculpteur du marbre, savoir son utilisation en quantité juste dans chaque partie de l'œuvre, celle-là est vertueuse dans la mesure où elle est la manifestation de la quantité juste ${ }^{26}$ de sa matière propre, le pathos. Et cette quantité est adéquate relativement à nous, savoir qu'elle n'est pas une ni la même pour tous. Afin d'éviter l'identification avec la moyenne arithmétique (le milieu de la chose) que sa formule suggère, Joachim assimile la quantité juste à une moyenne personnelle (ce qu'est la médiété pros hemas),

23. Mentionnons deux autres difficultés : Urmson identifie comme matière de la vertu les affects seuls, alors que les deux Éthiques soulignent à maintes reprises que la vertu porte à la fois sur les affects et les actions (1104b14, 1106b15, 1106b25, 1107a5; 1220a32, 1222a12). De plus, le fait qu'Urmson parle de l'émotion au singulier montre qu'il rattache un seul affect à chaque vertu, mais Aristote spécifie, au début de certains exposés concernant les vertus particulières, une double matière, par exemple la crainte et l'intrépidité pour le courage $(1106 \mathrm{~b} 35,1228 \mathrm{a} 37)$, le plaisir et la peine pour la tempérance (1117b25-26, 1230b10), le don et l'acquisition pour la générosité (1120b27-28, 1231b28-29).

24. Urmson 1973, p. 226.

25. Idem.

26. C'est-à-dire right amount, Joachim 1951, p. 86 ; cette expression est récurrente dans son commentaire. En p. 88 et 91, il remplace amount par intensity, et mentionne degree en p. 90 ; toutefois, récapitulant son point de vue à la fin du chapitre, il revient à l'expression initiale, et insiste sur la notion de quantité, p. 94. 
variant selon les circonstances individuelles ${ }^{27}$, lesquelles comprennent le temps, les personnes, le motif et la manière, selon le passage 1106b21-23. Ainsi, la médiété ou quantité juste de pathos est proportionnelle à toutes ces considérations. Joachim définit la quantité à l'aide de l'idée de proportion, lieu commun de la philosophie grecque. La proportion se comprend, selon lui, comme le plan suivant lequel les matériaux s'agencent pour constituer un tout ; une œuvre réussie implique la quantité adéquate de matière, c'està-dire une matière proportionnée dans les moindres détails, dont on ne peut rien retrancher ni ajouter (1106b10). La proportion, autrement dit, est la forme qui détermine la matière en quantité, qui fixe la quantité juste d'émotion à manifester pour produire la vertu ${ }^{28}$.

Les interprétations quantitatives offertes par Urmson et Joachim présentent des similitudes concernant l'identification de la médiété avec la quantité adéquate d'émotion et la relation de la médiété avec la situation de l'agent. On constate par ailleurs leur commune conception de l'affection en tant que matière de la vertu, en quoi ils visent juste ; Aristote est constant sur cette donnée: les états mettent en jeu des affections (1105b25, 1220b18-20). Ces ressemblances d'une part indiquent que le commentaire le plus récent s'inspire du plus ancien, et d'autre part rendent les textes susceptibles de la même critique concernant la compréhension de la médiété qu'ils proposent. Bien que le commentaire de Joachim soit plus précis, aucun des deux interprètes n'explique comment la notion de quantité s'applique à la vertu morale. De plus, en misant sur l'idée de right amount, les deux auteurs se rapprochent d'une interprétation dont l'inexactitude est démontrée, par Urmson lui-même du reste (voir la section précédente). Il s'agit de l'interprétation définissant la vertu en termes de modération, comme un état visant les affects moyens ou actions moyennes, et non comme un état moyen mettant en jeu des affects et des actions. En effet, écrire, comme Urmson, que la vertu consiste à être disposé de façon à manifester l'émotion en jeu selon la quantité adéquate peut signifier un affect moyen. L'objection s'applique pareillement à Joachim quand il affirme que la vertu, ou bonne action, réside dans la manifestation de la quantité juste d'émotion.

Malgré ces difficultés, la position d'Urmson demeure convaincante aux yeux de certains commentateurs ${ }^{29}$; d'autres, en revanche, s'emploient à dé-

27. Joachim 1951, p. 86, mais il précise ensuite que cette moyenne personnelle se situe tout de même à l'intérieur de certaines limites fixées par la nature humaine, p. 88 .

28. Ibid. p. 93-94,

29. Voir par exemple Hutchinson 1995, p. 217 sqq. et Garver 2006, p. 111-112, qui reprennent l'idée du right amount sans la problématiser, et Curzer 1996, qui défend sa plausibilité. 
montrer l'insuffisance de l'interprétation quantitative ${ }^{30}$. Deux de ces détracteurs prennent pour cible le fondement de l'explication urmsonienne de la vertu, à savoir le concept de right amount. D'abord, Rosalind Hursthouse remarque que la vertu, se définissant contrairement au vice (ni trop ni trop peu: 1106a33), constitue, dans une optique quantitative, une disposition à manifester la quantité adéquate d'émotion selon les mêmes paramètres que le vice (objet, occasion, façon, motif). Or, souligne l'auteure, comprendre le motif de la vertu en tant que quantité adéquate entre trop et trop peu de motifs est en soi absurde ${ }^{31}$. Il en va de même pour l'objet, autre paramètre ne pouvant constituer une quantité juste entre trop et trop peu. Par exemple, dans le cas du courage, craindre l'objet que l'on doit n'a rien à voir avec le fait de craindre un nombre $x$ d'objets qui serait une médiété entre trop et trop peu d'objets; il s'agit plutôt de craindre les objets appropriés, la mort et la souffrance ${ }^{32}$. D'après Hursthouse, beaucoup de vices s'analysent de la sorte, leur essence ne consistant pas à s'exercer sur un nombre trop grand ou insuffisant d'objets, mais sur des objets inappropriés. Cette explication permet à l'auteure de reconnaître la complexité à l'œuvre dans la description des travers donnée par les deux Éthiques et ainsi d'associer plusieurs états extrêmes à une seule vertu (autant qu'il y a de paramètres impliqués); elle donne l'exemple des multiples vices par excès associés à la colère (1126a9 sqq.) - ajoutons le cas emblématique de l'avarice, qui présente plusieurs formes (signalées en 1121 b16 sqq.; 1232a10-15). Ce faisant, elle offre une alternative pertinente au schéma triadique traditionnel excès/médiété/défaut dont Urmson se fait le porte-parole.

L'aspect réducteur du type d'interprétation strictement quantitative qui émerge des objections de Hursthouse est également dénoncé par Peter Losin, qui utilise un argumentaire comparable. Ayant rapporté l'essentiel de la doctrine de la médiété telle que présentée au début du livre II de l'EN,

30. Dans l'idée peut-être que la distinction entre le bien et le mal - entre la vertu et le vice - n'est pas seulement une question de quantité, comme le notait déjà Burnet 1900, p. 70.

31. Hursthouse 1980, p. 61. Il est exact que la vertu n'est pas affaire de nombre de motifs; en fait, la vertu a un seul motif, toujours le même, la beauté morale. Aristote est particulièrement clair à cet égard lorsqu'il traite du courage $(1115 \mathrm{~b} 13,1115 \mathrm{~b} 23,1116 \mathrm{a} 11$ 12, 1116b3, 1116b30, 1117a17, 1117b9, 1229a4, 1230a33). Il est par ailleurs exact que le motif de la vertu n'est pas quantifiable, cependant la critique de Hursthouse n'a ici aucune portée dans la mesure où le motif, plutôt qu'un paramètre, constitue un critère de vertu; la beauté morale est vraisemblablement ce qui fait de la vertu un sommet (1107a7, 1107a23), et ce qui explique sa grandeur (1169a18-35). Notons aussi que la stratégie de Hursthouse est plutôt insidieuse, car elle profite de sa critique contre Urmson pour remettre en question la doctrine aristotélicienne de la médiété, comme si cette dernière se réduisait à l'interprétation qu'en donne Urmson.

32. Ibid.p. 67 ; voir 1115a32-35; 1229b13-17. 
l'auteur signale son accord avec Hursthouse : l'interprétation quantitative d'Urmson constitue une simplification du concept de médiété pros hemas ${ }^{33}$. Losin affirme avec justesse que l'exégèse du right amount mène Urmson sur une fausse piste. En effet, ce dernier, misant prioritairement sur les notions de trop et de trop peu, résume la médiété en une quantité entre ces deux extrêmes ${ }^{34}$; autrement dit, Urmson ne prend pas garde au fait que l'agent courageux (vertueux) évite également de craindre des objets inappropriés, d'une façon et à un moment inadéquats, et que toutes ces attitudes à fuir ne se réduisent pas à une crainte trop intense (ni au fait de craindre un trop grand nombre d'objets). Bien entendu, craindre indûment implique le fait de craindre trop (c'est le travers du lâche, 1107b3-4, 1228b6, b37), mais le vice ne s'y réduit pas. Afin de le montrer, Losin développe de manière optimale (et charitable) l'analyse quantitative, en appliquant le modèle d'excès et de défaut à tous les paramètres impliqués par la médiété, soit la fréquence (jamais/toujours), le degré (trop doucement/trop violemment), la durée (trop court/trop long), les objets (personne/tout le monde) et les circonstances (rien/tout) ${ }^{35}$. Les affects et actions, matière de chaque vertu, peuvent se situer n'importe où sur chacun de ces cinq continuums, lesquels sont indépendants les uns des autres et expliquent que plusieurs vices correspondent à une seule vertu. Poussée à bout, l'interprétation quantitative révèle d'une part l'aspect limitatif du schéma triadique qu'adoptent pourtant plusieurs de ses défenseurs, et d'autre part l'incapacité dans laquelle elle se trouve de rendre compte de l'aspect d'inconvenance inhérent aux extrêmes. Par exemple, se mettre en colère à un moment inopportun n'est pas exclusivement affaire de l'être trop souvent, non plus que se mettre en colère contre les mauvaises personnes signifie invariablement contre trop de personnes (et ainsi de suite pour les autres paramètres pour chacune des vertus). Mais il faut convenir que la description quantitative de la médiété existe bel et bien - le discours de l'excès et du défaut est proprement aristotélicien, présent dans les traitements eudémien et nicomachéen de la vertu morale $^{36}$; impossible, dans cette perspective, d'en faire abstraction ${ }^{37} . \mathrm{Et}$

33. Losin 1987, p. 329-333.

34. Losin 1987, p. 333 ; en fait, l'auteur assimile l'interprétation quantitative à celle de la modération, p. 336 .

35. Losin 1987, p. 335. L'auteur dégage ces paramètres sur la base de l'exposé nicomachéen sur la douceur (1125b27-1126a29); signalons qu'ils apparaissent aussi, de façon générale, en $1106 \mathrm{~b} 22$ et en 1109a28.

36. Il fait même partie de la définition de la vertu morale en EN 1107a2-5, et il s'illustre dans le tableau eudémien des états particuliers (1220b38 sqq.).

37. Comme voudrait le faire Hursthouse 2005, dans une contribution où elle propose une interprétation « centrale » de la doctrine de la médiété, qui se fonde sur l'analogie établie par Aristote entre la vertu et le milieu d'un cercle (1109a25), tous deux difficiles à 
même si l'exégèse quantitative reste discutable au regard de certains éléments, il faut reconnaître qu'elle comporte une part de vérité : certes elle s'applique moins aisément aux paramètres tels que l'objet et le temps, néanmoins le paramètre d'intensité est quantitatif de manière intrinsèque ${ }^{38}$. En effet, il semble inconcevable de craindre une chose avec une intensité inappropriée sans la craindre trop ou trop peu, sans se trouver dans l'excès ou le défaut par rapport à l'affect en question; sous ce rapport, il convient tout à fait de s'exprimer en des termes quantitatifs.

En fait, pour tirer parti adéquatement de l'interprétation quantitative, il faut voir dans quelle mesure la notion de quantité s'applique à la vertu morale. Signalons d'emblée que la vertu, en tant que médiété ou milieu, relève bel et bien du domaine de la quantitée $e^{39}$. Mais il y a quantité et quantité d'après Métaphysique, $\Delta, 13$ : si elle se compte, se calcule, la quantité est multiplicité ; si elle se mesure, la quantité est grandeur (1020a8-10; voir aussi Catégories, 4b20 sqq.) - autrement dit, la quantité est soit discrète, soit continue. Or dans les deux Éthiques, Aristote situe la vertu dans le registre de la quantité continue (1106a26, 1220b21), prenant soin dans l'EN d'écarter le milieu conforme au rapport arithmétique (le milieu de la chose [1106b7], qui est une quantité discrète). Ainsi, même si la vertu n'est pas une quantité fixe ${ }^{40}$, la moyenne par rapport à nous n'est pas non plus une mesure indéfinissable; n'étant pas une ni la même pour tous (1106a33), la moyenne déterminée relativement à nous n'en demeure pas moins une mesure précise et objective ${ }^{41}$, comme en témoigne l'exemple de Milon, en 1106b1-5. Le diététicien, dans ce cas, prescrira une moyenne propre à chacun des deux intéressés; bien que différentes, toutes deux seront une mesure exacte de nourriture. De même, la vertu, se caractérisant comme une moyenne, appartient au registre des quantités (continues) qui se déterminent relativement; comme le plus, le moins et l'égal, l'excès, le défaut et le milieu se disent relativement les uns aux autres. Dans l'EE (1220b20 sqq.), traitant de la quantité continue, Aristote reconnaît que le milieu peut être pros allela, puisqu'il se dit par rapport aux autres termes de la série ${ }^{42}$.

atteindre. Selon elle, la «médiété-centre-de-la-cible » permettrait de rejeter comme déformant le discours de l'excès et du défaut (p. 108).

38. Pearson 2006, p. 89, 90.

39. Delabays 1946, p. 19, citant le commentaire d'Eustrate.

40. Comme l'observe à propos Pakaluk 2005, p. 112. Aristote prévient d'ailleurs, au début de son examen nicomachéen de la vertu morale, qu'il faut en cette matière renoncer à la rigueur étant donné que les biens dont se réclament nos actions n'ont rien de fixe (1104a15 , voir encore 1094b14-19).

41. Burger 2008, p. 57 ; Natali 2010, p. 85.

42. Plutôt que de contraster le milieu par rapport à nous au milieu de la chose comme le fait l'EN (1106a29-36), l'EE signale que l'excès, le défaut et le milieu peuvent se dire l'un par rapport à l'autre; ce mode de relation est tout à fait pertinent pour la description de la 
Pour saisir correctement la vertu, il est donc nécessaire de reconnaître des relatifs au sein de la quantité, et de prendre acte du statut ambigu des grandeurs relatives ${ }^{43}$; la vertu, à titre de quantité relative, peut être traitée d'une façon ou de l'autre, suivant la perspective qu'on adopte. Le défaut des interprétations quantitatives n'est donc pas tant d'être inexactes que de présenter une explication partielle de la nature de la vertu. En réalité, ces exégèses se concentrent sur ce que la vertu, en tant qu'état, a pour matière

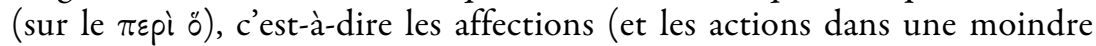
mesure, bien qu'il s'agisse là d'une erreur). Cette optique est tout à fait légitime, surtout considérant l'EE: les hypothèses qui y sont formulées au sujet de la vertu morale incluent une précision concernant la matière que celle-ci met en jeu. En plus de la définir comme une médiété, Aristote y mentionne que la vertu morale met en jeu certains milieux ( $\tilde{\omega}_{\sigma} \tau^{\prime} \dot{\alpha} \nu \dot{\alpha} \gamma \kappa \eta$

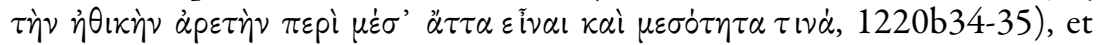
qu'elle a pour matière un certain milieu (dans le plaisir et la peine : $\pi \varepsilon p i$

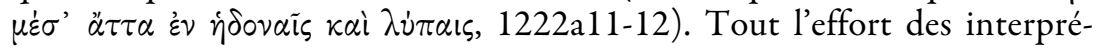
tations quantitatives est donc là : donner la mesure du milieu dans les affections et les actions, matière de la vertu morale, à l'aide des paramètres dont elles sont susceptibles - chaque paramètre précisant une variable quantifiable selon le trop ou le trop peu sur le continuum que supposent affect et action. Ce genre d'enquête, éclairant sous le rapport de la grandeur, ne dit rien cependant de la dimension pros hemas de la vertu morale, savoir qu'elle ne précise pas ce à quoi la médiété, en tant que relatif, est relative. L'examen des interprétations relativistes de la vertu morale, qui misent sur cette dimension, permettra de compléter le propos quantitatif.

\section{Les interprétations relativistes de la médiété pros hemas}

L'autre tendance exégétique, qui considère la vertu morale comme quantité déterminée relativement, rallie la plupart des commentaires récents. Plutôt que de s'intéresser à l'aspect quantitatif de la vertu, les commentateurs « relativistes » recherchent l'élément par rapport auquel elle est relative - autrement dit ce à quoi le milieu, en tant que relatif, est relatif (meson pros ti). Afin de départager ces nombreuses contributions, la distinction opérée par Stephen Leighton s'avère fort utile ; ce dernier, en effet,

médiété éthique, parce qu'il explique la variabilité de cette dernière. La médiété, dans cette optique, est fonction des autres termes de la série : elle se présente différemment selon la perspective d'après laquelle elle est considérée. En effet, tout comme l'égal est plus grand comparativement au plus petit, et plus petit comparativement au plus grand (1220b33), de même le milieu paraît excès par rapport au défaut, et défaut par rapport à l'excès. Ce type de milieu identifié par l'EE seule joue donc un rôle dans l'explication des rapports entre la médiété et les extrêmes.

43. Cf. Bodéüs 2001, n. ad Cat. 5b29, p. 113-114. 
identifie à juste titre deux stratégies au sein même de l'interprétation relativiste de la médiété pros hemas. Il nomme la première « relativité circonstancielle », laquelle consiste à rapporter la médiété à des affects et actions proportionnés par leur relativité aux circonstances (dans lesquelles la médiété est requise). Ce point de vue, explique Leighton, évacue la question du sens à donner au pronom dans l'expression «par rapport à nous » (figurant dans la définition de la vertu morale), car seul l'examen des cas particuliers demeure pertinent. La vertu étant sensible aux seules circonstances (aucunement aux agents), différentes situations généreront différents comportements, et un même contexte ne pourra produire de réponses vertueuses divergentes ${ }^{44}$ (si tant est que deux agents différents puissent effectivement se trouver dans la même situation). La seconde stratégie est nommée « relativité du caractère »; la vertu, dans cette optique, est relative à l'identité de l'agent, à ce qu'il est, de sorte que les individus diffèrent dans leur rapport à la vertu tout en étant néanmoins pleinement vertueux ${ }^{45}$ - la question du « nous » conservant ici toute son importance.

Cela dit, les contributions de ce deuxième sous-groupe sont particulièrement sujettes aux écueils identifiés par L. Brown et répertoriés en première section de ce texte; les propos de Losin et de Leighton, de fait, finissent par donner dans cette « dérive individualiste ». Ainsi Losin, suite à sa critique bien inspirée de l'interprétation quantitative, assimile $\dot{\eta} \mu \tilde{\alpha} \varsigma$ à l'individu particulier (aux particularités de chaque individu). L'auteur introduit la variabilité au sein de la médiété sur la base du passage 1106b2933, où Aristote indique qu'il est facile de rater sa cible et difficile de l'atteindre et que c'est pourquoi le milieu constitue une réussite et appelle les louanges ${ }^{46}$. Prenant les termes $\sigma \tau 0 \chi \alpha \sigma \tau \kappa \kappa \dot{~ e t ~} \sigma \kappa o \pi \dot{\delta} \zeta$ très au sérieux ${ }^{47}$, Losin comprend par là que même si le fait de toucher son objectif exige plus de précision que de le manquer, le succès laisse place à la variation. Autrement dit, comme plusieurs tirs peuvent atteindre le centre de la cible et obtenir un score identique, l'analogie de la cible suggérerait que la vertu ne requiert pas une action ou un affect unique d'une intensité, durée et fréquence fixes, mais davantage une action ou un affect situé à l'intérieur d'un intervalle plus ou moins précisément circonscrit de possibilités ${ }^{48}$. Et cette variation

44. Leighton 1995, p. 72.

45. Ibid. p. 69.

46. Il importe de noter que ce passage n'a pas de parallèle dans l'EE. Le fait est que l'EE se préoccupe assez peu de l'aspect laudatif de la vertu; elle demeure ainsi dans un registre plus neutre que l'EN, qui fait la promotion de la vertu en spécifiant à plusieurs reprises qu'elle vaut des louanges à son possesseur (voir entre autres 1106a1, 1106b29, 1109a30).

$47.1106 \mathrm{~b} 15,28,32$; aucune trace de l'analogie de la cible dans l' $E E$; Aristote n'insiste pas, dans cet ouvrage, sur la difficulté d'atteindre le milieu.

48. Losin 1987, p. 331-332. 
est fonction de « nous », à savoir de chaque individu ; puisque chaque personne est distincte, la médiété relative à nous le sera aussi, et se déterminera en rapport avec les attributs de la personne concernée et des circonstances dans lesquelles elle se trouve. Pour Losin donc, la médiété est relative et à l'agent et aux circonstances : à l'agent d'une part, car il est impossible d'établir si un tel est ou n'est pas courageux sans savoir ce qui personnellement lui inspire crainte et intrépidité, et aux circonstances d'autre part, car atteindre la cible implique, pour l'agent, le fait d'être conscient de l'ensemble des facteurs composant la situation et de $s^{\prime} y$ adapter ${ }^{49}$. Losin considère que pour fixer la médiété dans un cas particulier, il importe de tenir compte des caractéristiques de ce cas, et que parmi celles-ci se trouve la personnalité de l'agent ${ }^{50}:$ la constitution émotionnelle de chacun influence donc la réussite ou l'échec, et demande divers degrés d'adaptation. Si l'identification que Losin établit entre « nous » et la sensibilité affective de l'individu s'apparente à la deuxième dérive dénoncée par Brown (voir supra, p. 245-246), sa contribution met pourtant en lumière un élément important à l'aide de l'analogie de la cible : la médiété éthique se situe à l'intérieur d'un ensemble plus ou moins précisément circonscrit de possibilités. Certes la variabilité n'est pas fonction de la constitution émotionnelle de chacun, néanmoins, n'étant pas une chose unique ni la même pour tous (1106a32), la médiété constitue l'un des choix possibles sur un continuum de comportements éventuellement vertueux. Telle est la position de Carlo Natali ${ }^{51}$, qui explique que la médiété n'est pas conçue par Aristote comme un point sur une ligne, mais comme un segment qui comprend une série de comportements vertueux. L'auteur se base sur 1109b18-23, où le Stagirite signale que ceux qui s'éloignent un peu du bien ne sont pas blâmés, étant donné la difficulté de déterminer la médiété.

Il est cependant possible de défendre la relativité du caractère de façon plus efficace que Losin ; Lesley Brown et Paula Gottlieb y arrivent toutes deux en tirant argument de l'analyse des vertus morales particulières (plutôt que de la théorie de la vertu morale). Bien que la première soit en fait partisane de la relativité circonstancielle, elle souligne la pertinence de certaines caractéristiques de l'agent dans la détermination de la vertu; par exemple, la générosité est fonction de la richesse de l'agent, car le don s'exerce en rapport avec l'avoir, de sorte que celui qui donne moins peut être jugé plus généreux si son avoir est moindre ${ }^{52}$. Dans le même ordre

49. Losin 1987, p. 332 et 337.

50. Ibid.p. 338.

51. Natali 2001, p. 36-37. L'idée est reprise dans Curzer 2012, p. 53.

52. Brown 1997, p. 86, citant 1120 b10 (sans parallèle dans l'EE). 
d'idées, Gottlieb défend sa position ${ }^{53}$ à l'aide du cas de la générosité, parce qu'il est le plus clair. Aristote remarque en effet que cette vertu est relative aux moyens de la personne qui donne ; la marque du généreux ne se trouve pas dans la quantité de biens donnés, mais dans l'état du donateur, ce qui explique qu'on peut juger plus généreux l'individu qui donne moins, s'il donne à partir de moins (1107b7-11). Comme la générosité, la magnificence est relative au statut de l'agent: Gottlieb cite 1123b23-26, où le Philosophe affirme qu'on juge de la dépense en se rapportant à l'agent - on voit qui il est et quelles sont ses ressources (voir aussi 1233b10). La franchise et la magnanimité impliquent aussi une relativité à l'agent : pour être franc, l'agent doit reconnaitre ses propres avantages sans les diminuer ni les majorer (1127a24-26 ; de même, 1234a1-4) et pour être magnanime, il doit se connaître lui-même (voir 1123b2, 14-15, 31-32). Étant donné ces passages, difficile de nier que la vertu comporte une certaine relativité eu égard aux caractéristiques de l'agent - même si elle ne dépend pas de la personnalité de chacun. Il convient donc ici de rectifier légèrement la terminologie : mieux vaut parler de relativité eu égard aux caractéristiques de l'agent plutôt que de parler de relativité du caractère. La dernière expression, en effet, expose celui qui l'utilise à verser dans un subjectivisme qui ne correspond pas à la théorie de vertu morale exposée dans les Éthiques.

Même si Leighton, en liant le « nous » au caractère, expose son propos aux difficultés mentionnées ci-dessus (supra, p. 245), le traitement qu'il donne du fameux exemple de Milon mérite d'être examiné. Propre à l'EN, cet exemple illustre la notion de milieu relatif à nous-même par opposition au milieu de la chose; voici l'extrait :

Si pour un homme, dix mines à manger, c'est beaucoup et que deux, c'est peu, le diététicien ne va pas pour autant prescrire invariablement six mines, car c'est peut-être encore beaucoup pour celui qui doit les prendre, ou bien trop peu. Pour Milon, en effet, c'est peu, mais pour qui débute en gymnastique, c'est beaucoup. (1106b1-4.)

Selon Leighton, ce passage sert à la fois le parti de la relativité du caractère et celui de la relativité circonstancielle, preuve de leur compatibilité. Dans cette dernière optique, l'entraîneur (le diététicien) détermine la quantité moyenne de nourriture en fonction des conditions de vie de l'athlète, de la nourriture disponible, de la nature et du moment de sa prochaine épreuve, etc., toute variation de ces facteurs entraînant une modification de la médiété. Or une telle compréhension du texte, note Leighton,

53. Gottlieb 2009, p. 25-32 (troisième section de la première partie, intitulée « The mean is 'relative to us' »). 
n'exclut pas la première stratégie ${ }^{54}-$ les deux sont en fait complémentaires, de sorte que l'exemple de Milon reflète aussi l'importance du caractère dans la doctrine de la médiété. Leighton souligne à cet effet qu'Aristote luimême n'invoque pas les conditions propres au fameux athlète; il se limite à mentionner Milon, démontrant ainsi que ce qui convient se détermine en fonction de cet agent-ci, de sa nature propre ${ }^{55}$. Mais voilà, le propos de Leighton comporte un glissement dans l'explication de l'exemple : alors que dans l'optique de la relativité circonstancielle, l'agent moral correspond à l'entraîneur, dans l'optique de la relativité du caractère, il correspond à Milon. Pourtant, selon les termes mêmes de l'EN, ce n'est pas Milon luimême qui détermine le régime, mais le diététicien. Brown argumente précisément dans ce sens : l'exemple de Milon, souligne-t-elle, met en jeu un seul agent moral, l'entraîneur, qui fixe deux médiétés, l'une pour Milon, l'autre pour le débutant. Et celles-ci ne varieront pas en fonction de l'agent moral - pas plus que la diète appropriée ou le médicament convenable ne sont fonction du diététicien ou du médecin qui les prescrit ${ }^{56}$. En d'autres termes, le meilleur régime (la médiété) différera selon des facteurs contextuels, et non selon le nutritionniste (l'agent moral). Or l'exemple de Milon peut encore se comprendre de la façon suivante: Aristote illustrerait au moyen de deux individus ce qui en réalité se produit en un seul afin de montrer que la vertu diffère d'une personne à l'autre dans la pratique tout en demeurant formellement la même. Dans les deux cas, pour Milon et pour le débutant, le même entrâneur fixe le meilleur régime qui se traduit différemment dans les faits ; pareillement, dans chaque cas particulier, l'excellence morale reste la même - elle est un sommet de perfection $(1107 \mathrm{a} 7,23)$ - en se concrétisant de façon variable d'un agent moral vertueux à l'autre. Ainsi l'exemple de Milon, mettant en relief l'importance des caractéristiques de l'agent et de la situation dans laquelle il se trouve, montre que la médiété pros hemas ne se détermine pas une fois pour toutes, de manière abstraite, en dehors de tout contexte (individuel et circonstanciel), mais demeure à déterminer à chaque fois que la vertu morale est requise. Dans cette perspective, Aristote, en nommant Milon, signale à l'avance que la définition de la vertu morale qu'il s'apprête à donner est, de par sa généralité, insuffisante ${ }^{57}$, ce qui du coup explique la nécessité du catalogue des vertus morales occupant les livres III et IV de l'EN, lesquels servent à appliquer cette caractérisation, à la déployer à tous les cas particuliers ${ }^{58}$.

54. Leighton 1995, p. 74.

55. Ibid.p. 75.

56. Brown 1997, p. 87-88.

57. Les définitions universelles sont qualifiées de creuses en 1107a28-32.

58. Natali 2010, p. 84 et 87. 
Dans la mesure où chacune des deux sous-catégories d'interprétation relativiste concède à l'autre (les défenseurs de la relativité circonstancielle admettent que certains attributs de l'agent doivent être considérés, et les partisans de la relativité du caractère conviennent que la situation est un facteur dont il faut se préoccuper), l'idée d'ordonner les contenus exacts de l'une et de l'autre s'impose naturellement à qui recherche une compréhension adéquate de la vertu morale. Ce procédé fructueux est adopté par Ronna Burger qui, dans son commentaire de l'EN, rappelle fort à propos que selon Aristote, le bien est toujours le bien pour quelqu'un dans une situation particulière; la vertu morale, considérant ce fait, consiste en la réponse convenable, dans l'affect et l'action, d'un individu particulier dans une situation particulière ${ }^{59}$. Cette importante observation peut être rattachée à la critique de l'idée du Bien, présente au début des deux Éthiques, où Aristote s'emploie notamment à en dénoncer la trop grande généralité. Le bien idéal, en admettant même qu'il existe, s'avère inutile; il est évident, note Aristote, qu'il ne serait pas à la portée de l'être humain, ce qui le rend inexécutable. Or il s'agit justement du type de bien que vise la quête éthique, ce qu'éclaire le parallèle avec la médecine : comme le médecin ne considère pas « la » santé, mais celle de l'être humain, voire de cette personne-ci - il soigne le particulier -, pareillement l'agent moral ne recherche pas «le» bien, mais le bien humain, celui qui est exécutable (1096b321097 a14). Le bonheur, précise l'EE, est la meilleure des bonnes choses à la portée de l'action de l'être humain $(\tau \tilde{\omega} \nu \dot{\alpha} \nu \theta \rho \dot{\omega} \pi \omega) \pi \rho \alpha \kappa \tau \tilde{\omega} \nu, 1217 \mathrm{a} 40$, 1218b12). Dans la perspective de cette comparaison médicale, le bien, exécutable par l'agent moral, rapporte la vertu morale à ce dernier ; les circonstances, quant à elles, déterminent la particularité des actes de l'agent. Ainsi deux éléments - complémentaires et non concurrents - se distinguent au sein de la médiété pros hemas : ce à quoi elle est relative et ce qui la particularise. En tant que bien exécutable, la vertu est d'abord et avant tout vertu d'un être singulier; elle est, pour reprendre l'expression eudé-

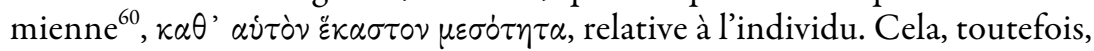
ne signifie pas que la médiété est fonction de la constitution affective de chacun, comme il a été établi précédemment. Moyenne relative à l'agent, la vertu se concrétise par le biais de la situation; autrement dit, parmi toutes les caractéristiques de l'individu, celle qui particularise ses actes est le contexte dans lequel il se trouve - c'est sans doute pourquoi nombre de commentateurs écrivent, de façon un peu lapidaire, que la médiété est fonc-

59. Burger 2008, p. 58-60.

60. Voir 1222a10-11(Susemihl 1884); signalons toutefois qu'il y a litige à propos de ce passage. 
tion des circonstances ${ }^{61}$. Une telle singularité explique qu'il n'existe pas de procédure fixée à l'avance pour la déterminer ${ }^{62}$. L'agent vertueux n'est pas disposé à ressentir et à agir d'une manière routinière ; au contraire, les circonstances qui informent ses actes demandent de sa part une certaine flexibilité $^{63}$. D'ailleurs l'EN est claire à ce sujet : les biens dont se réclament nos actions n'ont rien de fixe (1104a4; voir aussi 1094b14-16), aussi les agents doivent tenir compte des circonstances opportunes (1104a8). Celles-ci déterminent l'aspect concret et à chaque fois singulier de la médiété, et sont en même temps ce qui échappe à la définition (ou à l'argumentation) rigoureuse $^{64}$. La situation, toujours nouvelle, toujours autre et ne se répétant jamais, impose ses exigences au comportement vertueux, lequel, au demeurant, reste indéfinissable ${ }^{65}$. Là se trouve toute la difficulté de la vertu : les cas particuliers impliquent une multitude de données sensibles qu'on ne peut définir (1109b21-24), expliquant que le bien soit chose rare, louable et belle (1109a29-30).

De telles considérations sur le particulier nous ramènent à un important constat soulevé par l'interprétation quantitative, celui de la multiplicité des paramètres à observer eu égard à la matière mise en jeu par la vertu morale. Affections et actions, en effet, sont susceptibles de plus et de moins selon diverses modalités dont la réalisation particulière relève des circonstances. À leur façon donc, les deux courants interprétatifs, quantitatif et relativiste, rendent compte d'une donnée essentielle concernant la vertu du caractère : sa nature multidimensionnelle. Une telle richesse fait apparaitre toute la densité des formules de 1107al et de 1227b7. Si le genre se comprend comme matière de la différence ${ }^{66}$, la vertu en tant que médiété pros hemas c'est-à-dire médiété relative à l'agent - a pour matière ce que précisément elle met en jeu en tant qu'état, savoir actions et affections, matière dont les paramètres varient en regard des circonstances dans lesquelles l'agent se trouve. Génériquement hexis peri ti et spécifiquement mesotes pros $t i$, la

61. Hardie 1965, p. 188 ; Urmson 1988, p. 35 ; Hutchinson 1995, p. 217 ; Brown 1997, p. 86 ; Bostock 2000, p. 41 ; Natali 2001, p. 36 ; Achtenberg 2002, p. 116, 122 ; Kontos 2002, p. 74 ; Hursthouse 2005, p. 104-105; Wang 2007, p. 413, 417-418.

62. Losin 1987, p. 337 ; Lawrence 2009, p. 429.

63. Achtenberg 2002, p. 118, 121. Rappelons à cet égard que la médiété éthique se distingue du milieu arithmétique; parfois, elle est plus près de l'excès (dans le cas du courage et de la générosité), parfois du défaut (dans le cas de la tempérance). Cf. 1109a1-5; $1234 \mathrm{~b} 10-13$.

64. Schilling 1930, p. 42. La même rigueur ne doit pas être réclamée dans tous les arguments; voir supra, n. 40.

65. Le particulier concret est indéfinissable (Métaphysique, Z, 15, $1039 \mathrm{~b} 28$ sqq.); il n’y a pas de définition de ce qui peut être autrement, et l'exécutable se trouve justement dans ce cas (cf. 1112a30-b9).

66. Metaph. $\Delta, 28,1024 \mathrm{~b} 9$. 
vertu morale aristotélicienne présente le statut particulier, bivalent, de grandeur relative, lequel autorise un traitement sous l'un ou l'autre de ces aspects. 


\section{BIBLIOGRAPHIE}

ACHTEnberg, Deborah 2002: Cognition of Value in Aristotle's Ethics: Promise of Enrichment, Threat of Destruction, Albany, 2002.

BLOCH, Olivier \& Antoine LÉANDRI 2011 : Aristote, Éthique à Eudème. Introduction, traduction et notes, Paris, 2011 (Encre marine).

BODÉÜs, Richard, 2001 : Aristote, [Catégories]. Texte établi et traduit par R. B. Paris, 2001 (Collection des Universités de France).

- 2004: Aristote, Éthique à Nicomaque. Introd. trad. et notes, Paris, 2004 (GFFlammarion 0947).

BOSLEY, Richard, 1991 : On Virtue and Vice : Metaphysical Foundations of the Doctrine of the Mean, New York, 1991 (Revisioning Philosophy, 6).

Bostock, David, 2000 : Aristotle's Ethics, Oxford, 2000.

BROWN, Lesley 1997: «What is the 'Mean relative to us' in Aristotle's Ethics? », Phronesis, 42 (1997), p. 77-93.

Burger, Ronna 2008: Aristotle's dialogue with Socrates: on the Nicomachean Ethics. Chicago, 2008.

BURNET, John 1900 : The Ethics of Aristotle, edited with Introd. and Notes, Londres, 1900.

CURZER, Howard J. 1996: «A Defense of Aristotle's Doctrine that Virtue is a Mean », Ancient Philosophy, 16 (1996), p. 129-138.

- 2012: Aristotle and the Virtues, Oxford, 2012.

DÉCARIE, Vianney 1978 : Aristote, Éthique à Eudème. Introduction, traduction, notes et indices, avec la collab. De R. Houde-Sauvé, Paris/Montréal, 1978.

Delabays, Georges 1946: La notion de milieu dans la philosophie pratique d'Aristote (Thèse), Fribourg (Suisse), 1946

DesLAURIERS, Marguerite 2003 : « Aristotle on the virtues of slaves and women », Oxford Studies in Ancient Philosophy, 25 (2003), p. 213-231.

EVRIGENIS, Ioannis D. 1999 : « The doctrine of the mean in Aristotle's ethical and political theory », History of Political Thought, 20 (1999), p. 393-416.

Garver, Eugene 2006: Confronting Aristotle's Ethics: Ancient and Modern Morality, Chicago, 2006.

GAuthier, René-Antoine \& Jean-Yves Jolif 1970: Aristote, L'Éthique à Nicomaque. Introduction, traduction et commentaire ( $2^{\mathrm{c}}$ éd. avec une introd. nouv.), Louvain/Paris, 1970 (Aristote : traductions et études).

GotTlieb, Paula 2009: The Virtue of Aristotle's Ethics, Cambridge/New York, 2009.

HARDIE, W. F. R. 1964-1965 : «Aristotle's Doctrine that Virtue is a 'Mean' », Proceedings of the Aristotelian Society, 65 (1964-1965), p. 183-204.

HÖFFE, Ottfried (éd.) 2010 : Aristotle's "Nicomachean Ethics", Leyde/Boston, 2010. [Trad. par D. Fernbach de: Aristoteles, Nikomachische Ethik, 2., bearb. Auflage, Berlin, 2006 (Klassiker auslegen, 2).]

Hursthouse, Rosalind 1980-1981: «A false doctrine of the mean », Proceedings of the Aristotelian Society, 81 (1980-1981), p. 57-72.

- 2005 : «The Central Doctrine of the Mean », dans R. Kraut (éd.), The Blackwell Guide to Aristotle's Nicomachean Ethics, Malden, MA, 2005 (Blackwell Guides to Great Works, 4), p. 96-115.

Hutchinson, D. S. 1995 : «Ethics », dans J. Barnes (éd.), The Cambridge Companion to Aristotle, Cambridge, 1995, p. 195-232.

JoACHim, H.H. 1951: Aristotle. The Nicomachean Ethics. A commentary by the late H. H. Joachim. Ed. by D. A. Rees, Oxford, 1951.

KenNY, Anthony 2011 : Aristotle, Eudemian Ethics. Translated with an Introduction and Notes, Oxford, 2011. 
KONTOS, Pavlos 2002: L'Action morale chez Aristote: une lecture phénoménologique et ses adversaires actuels, Paris, 2002 (Thémis. Philosophie).

LAWRENCE, Gavin 2009 : « Human Excellence in Character and Intellect », dans G. Anagnostopoulos, A Companion to Aristotle, Chichester, U.K./Malden, MA, 2009 (Blackwell Companions to Philosophy), p. 419-441.

LeIGHTON Stephen R. 1995: «The Mean relative to us », Apeiron, 28 (1995) = Aristotle, Virtue and the Mean, p. 67-78.

LORENZ, Hendrik 2009: «Virtue of character in Aristotle's Nicomachean Ethics », Oxford Studies in Ancient Philosophy, 37 (2009), p. 177-212.

LosIn, Peter 1987 : « Aristotle's doctrine of the mean », History of Philosophy Quarterly, 4 (1987), p. 329-341.

Natali, Carlo 2001: The Wisdom of Aristotle, Albany, 2001 (SUNY Series in Ancient Greek Philosophy). [Trad. par G. Parks de La Sagezza di Aristotele, Naples, 1989 (Elenchos, 16).]

- 2010: «Particular virtues in the Nicomachean Ethics of Aristotle», dans R.W. Sharples (éd.), Particulars in Greek Philosophy: The seventh S. V. Keeling Colloquium in Ancient Philosophy, Leyde/Boston, 2010, p. 73-96.

PAKALUK, Michael 2005 : Aristotle's Nicomachean Ethics: an Introduction, Cambridge, 2005 (Cambridge introductions to key philosophical texts).

PEARSON, Giles 2006: «Does the fearless phobic really fear the squeak of mice "too much"? », Ancient Philosophy, 26 (2006), p. 81-91.

ReEve, Charles David Chanel 2012: Action, Contemplation, and Happiness : An Essay on Aristotle, Cambridge, 2012.

Rowe, Christopher J. 2002 : Aristotle, Nicomachean Ethics. Transl. (with historical introd.) by C. J. R., philosophical introd. and comm. by Sarah Broadie, Oxford, 2002.

Schilling, Harald 1930 : Das Ethos der Mesotes, Tübingen, 1930 (Heidelberger Abhandlungen zur Philosophie und ihrer Geschichte).

Suseminl, Franciscus 1884 : [Aristotelis Ethica Eudemia] Eudemii Rhodii Ethica recogn. F. S., Leipzig, 1884 (Bibliotheca scriptorum graecorum et romanorum Teubneriana). [Réimpr. Amsterdam, 1967].

TAYlor, Christopher C. W. 2006: Aristotle, Nicomachean Ethics. Books II-IV. Translated with a commentary, Oxford, 2006 (Clarendon Aristotle Series).

Urmson, J. O. 1973: «Aristotle's Doctrine of the Mean», American Philosophical Quarterly, 10 (1973), p. 223-230.

- 1988: Aristotle's Ethics, Oxford, 1988.

WALZER, Richard R. et Jean M. MINGAY 1991 : Aristotelis Ethica Eudemia rec. breuique adnotatione critica instruxerunt R. R. Walzer \& J. M. Mingay, praefatione auxit J. M. Mingay, Oxford, 1991 (Scriptorum Classicorum Bibliotheca Oxoniensis).

WANG, Huaiyu 2007 : « Mesotes, energeia, aletheia: discovering an Arian's thread through Aristotle's moral and natural philosophy », Epoché: A Journal for the History of Philosophy, 11 (2007), p. 409-420.

WARnE, Christopher 2006: Aristotle's Nicomachean Ethics: Reader's Guide, Londres, Continuum, 2006 (Continuum Reader's Guides).

Williams 1985 : Ethics and the Limits of Philosophy, Londres, 1985.

WOLF, Ursula 2010: «The sense of Aristotle's doctrine of the mean (Book II) », dans Höffe 2010, p. 69-88. [Version révisée de « Über den Sinn der aristotelischen Mesoteslehre », Phronesis, 33 (1988), p. 54-75.]

Woods, Michael 1982: Aristotle, Eudemian Ethics books I, II, and VIII. Transl. with a comm., Oxford, 1982 (Clarendon Aristotle Series).

Young, Charles M. 1996: «The doctrine of the mean », Topoi: An International Review of Philosophy, 15 (1996), p. 89-99. 